\title{
Estrategias metodológicas para el mejoramiento de la comprensión lectora de los casos clínicos en Inglés
}

\author{
Methodological strategies for the improvement of Reading comprehension \\ of clinical cases in English
}

Christian Eduardo Meza Fiallos. ${ }^{1}$, Luis Oswaldo Guadalupe Bravo. ${ }^{2}$, Marco Antonio Bravo Montenegro. ${ }^{3}$, David Antonio Ureña Lara. ${ }^{4}$

\begin{abstract}
Introduction: Forever English in medical classrooms has been limited to teaching as EFL English as a foreign language or ESL English as a second language but little emphasis is placed on terminology of texts or clinical cases in English. However, the need to understand medical texts in English in classrooms persists due to their importance and use in the professional life of the medical community. Objective: To improve the reading skills of clinical cases in the English language with the use of linguistic clues that help with their best and quick understanding. Methods: An educational intervention was carried out on two groups of students in the fifth semester of the medical career through tests that demonstrate the reading comprehension of clinical cases in English for later analysis and with the application of reading strategies and linguistic clues the comprehension of medical treaties can improve and students may be able to read faster giving importance to understanding. The first group was evaluated to check the reading comprehension without having used the reading strategies and language aids and the second group was taken the test using the English language strategies and clues. The data were tabulated and the results were presented to the students for later discussion. Results: There was a significant increase in the general understanding of the subject in clinical cases thanks to the students' understanding of the language aids present in the English

\footnotetext{
${ }^{1}$ Escuela Superior Politécnica de Chimborazo, Facultad de salud publica. carrera de medicina, Chimborazo, Ecuador, christian.meza@espoch.edu.ec

${ }^{2}$ Escuela Superior Politécnica de Chimborazo, Facultad de salud publica. carrera de medicina, Chimborazo, Ecuador, loguadalupe@espoch.edu.ec

${ }^{3}$ Escuela Superior Politécnica de Chimborazo, Facultad de salud publica. carrera de medicina, Chimborazo, Ecuador, marcoa.bravo@espoch.edu.ec

${ }^{4}$ Escuela Superior Politécnica de Chimborazo, Facultad de salud publica. carrera de medicina, Chimborazo, Ecuador, davidurenia@espoch.edu.ec
} 
language and the use of reading strategies. Conclusions: The present intervention helped to have knowledge of the reason why students in the medical career have difficulty with understanding medical texts in English and their reading strategies were improved giving a sense of the importance of using the English language linguistic clues.

Palabras claves: Clinic cases, linguistic clues, reading comprehension, reading strategies.

\section{Resumen}

Introducción: Por siempre el Inglés en las aulas de medicina se ha circunscrito a la enseñanza como EFL Inglés como lengua extranjera o ESL Inglés como segunda lengua pero se pone poco énfasis en la terminología de textos o casos clínicos en Ingles. Sin embargo, la necesidad de comprender textos de medicina en inglés en las aulas persiste debido a su importancia y uso en la vida profesional de la comunidad médica. Objetivo: Mejorar las destrezas lectoras de casos clínicos en el idioma Inglés con el uso de claves lingüísticas que ayuden con su mejor y rápida comprensión. Métodos: Se realizó una intervención educativa a dos grupos de estudiantes de quinto semestre de la carrera de medicina a través de pruebas que evidencien la comprensión lectora de casos clínicos en Inglés para su posterior análisis y con la aplicación de las estrategias de lectura y las ayudas lingüísticas se mejore la destreza de lectura de tratados médicos y se lea más rápido y mejor dando importancia a la comprensión. Al primer grupo se le tomó la prueba de comprensión lectora sin haber utilizado las estrategias de lectura y las ayudas lingüísticas y al segundo grupo se le tomo la prueba manejando y usando las estrategias y pistas lingüísticas del idioma Inglés. Se tabularon los datos y se presentó los resultados a los alumnos para su posterior discusión. Resultados: Se pudo constatar un aumento significativo de la comprensión general del tema en casos clínico gracias a la comprensión por parte de los alumnos de las ayudas lingüísticas presentes en el idioma Inglés y la utilización de estrategias de lectura. Conclusiones: La presente intervención permitió conocer la razón por la cual los alumnos en la carrera de medicina tienen dificultad con la comprensión de lecturas médicas y se mejoró sus estrategias de lectura dando a entender la importancia de utilizar pistas lingüísticas del idioma Inglés.

Palabras claves: caso clínico, pistas linguísticas, comprensión lectura, estrategias de lectura.

\section{Introducción}

El Inglés se ha convertido hoy por hoy en el lenguaje universal utilizado en todo aspecto de la vida del ser humano en todo el mundo. Desde utilizarlo para un viaje de turismo, para hacer una compra en línea o para estudio en el exterior.

Pero lamentablemente se ha estado quitando importancia al uso del Inglés en las universidades como formación de los estudiantes como herramienta principal para ahondar y profundizar en los temas de investigación y se ha relegado a dictar su catedra como EFL Inglés como idioma extranjero o ESL Inglés como segunda cuando lo imperioso es enseñar el idioma con un propósito académico lo que en lingüística se llama 
ESP (English for Specific Purposes), es decir enseñanza del Inglés para con propósitos académicos convirtiéndose así en un componente fundamental en el tronco común del currículo de la carrera de medicina.

Además, la importancia se incrementa cuando la mayoría de investigaciones se las encuentra en "papers" o artículos publicados sobre casos clínicos en todo el mundo por investigadores médicos pero la mayoría de estudiantes de medicina no están teniendo las herramientas suficientes para continuar con sus estudios de casos clínicos durante su carrera y después en su vida profesional.

Bosque menciona que el uso de una estrategia que permita desarrollar el proceso de formación del idioma Ingles con los estudiantes de medicina enfocado en la integración de habilidades receptivas como es en el caso de la lectura de casos clínicos en Ingles constituye una emergente solución a la problemática de la dificultad de comprensión de temas médicos cuando se lo hace en Inglés. [2]

Es importante notar que actualmente existen diversos programas informáticos en inglés que permiten a los estudiantes que se están formando en las aulas de medicina saber las palabras técnicas necesarias para su carrera sobre temas específicos cuando no se conoce alguna palabra en especial ya que no se puede esperar que se comprendan todas las palabras utilizando la estrategias de lectura y conociendo los componentes de lingüística si el contexto de la oración no es suficiente para su comprensión. [1]

Por eso, aunque existen ayudas informáticas como traductores y programas informáticos, eso nunca va a reemplazar al ser humano que debe procesar la información del L2 al L1 decodificando la información (input) a través del proceso lector para que sea entendible en su idioma. La mente nunca va a reemplazar a la maquina ya que el medico puede darle el sentido correcto de la información y no caer en una mala traducción de la máquina.

Con esto se puede entrever la importancia de la adquisición del vocabulario técnico y necesario en el idioma para tener una mejor comprensión del texto médico y la manera de lograrlo es a través de la lectura. Leer una variedad de textos es una parte esencial del crecimiento en vocabulario ya que se puede encontrar una cantidad de vocabulario de algunos géneros pero puede haber una carencia en otros. Por esta razón, se realizó la presente intervención en el campo médico con los alumnos de la carrera de medicina con el propósito de alzar y mejorar la comprensión lectora de textos médicos, PDFs y casos clínicos. [6] 
Una de la ayudas lingüísticas que los estudiantes tienen a su disposición son las "Collocations" esto sucede cuando dos o más palabras se juntan para dar un significado. En el campo de la medicina tenemos las siguientes: in "upset stomach" upset tiene que ir necesariamente con "stomach", no se puede usar ninguna otra palabra similar en Inglés para referirse a "dolor de estomago" como "malaise o discomfort" que individualmente significan lo mismo en español, o "blood test" lo cual significa examen de sangre y no se puede poner "blood exam". En tal virtud, cuando el estudiante se enfrente ante un texto médico, su lectura y su comprensión se hará más rápida y más comprensible. [5]

Laufer llego a la conclusión después de haber llevado a cabo sus estudios sobre "Cuanto léxico se necesita para la comprensión lectora" que cuando un estudiante se enfrenta a la interpretación en la lectura de un texto, primero recurre al significado de la palabra y después al conocimiento de la materia y pone poco énfasis en la sintaxis del texto. Esto lleva a concluir sobre la importancia de conocer los significados de las palabras y más aún las palabras técnicas de medicina ya que sin estos no se podrá decodificar el significado de las ideas expresadas en las oraciones del texto.

Además, saber el conocimiento de la materia en L1 es decir en Español no garantiza la comprensión general del texto en Inglés ya que es más importante el significa exacto de la palabra desconocida para pasar inmediatamente a la sintaxis para extraer el significado global de la oración. [7]

Spada menciona que la lectura consituye un manera unica de adquirir e incrementar la cantidad de vocabulary en L2 para estudiantes de segunda lengua de la misma forma como lo es para personas que aprenden el primer idioma. El problema empieza en las escuelas y colegio donde no se pone enfasis en la adquisition de palabras a traves de la lectura de textos en ingles y cuando los estudiantes van a la universidad se encuentran con pesimos niveles de comprension lector en general, y mas aun sobre libros, PDFs de casos clinicos. De ahí la importancia de dominar estrategias de lectura e identificar pistas linguisticas dentro de L1 y L2 (intralengua e interlengua) que permita el uso de tecnicas como asociación de palabras, colocaciones, morfologia de las palabras, gramatica, punctuation y significado de la oracion. [8]

\section{Métodos}

Se realizó un estudio experimental con el método cuantitativo con dos grupos de estudiantes de sexto año de la carrera de medicina en total 70 de la Universidad 
Politécnica de Chimborazo durante los meses de mayo y junio de 2019 a los cuales se les aplicó una prueba de comprensión lectora de un caso clínico relacionado con Cardiopatías para lo se investigó y escogió el mejor tema tomando en cuenta la materia Cardiología que tuvieron los alumnos en este ciclo de la carrera.

La prueba consistía en 40 preguntas del caso clínico con respecto a la diagnosis, tratamiento y recuperación de los pacientes con sus diferentes afecciones y síntomas sin haber discutido, enseñado y socializado con los alumnos las estrategias de lectura así como también las ayudas lingüísticas como "collocations, compound words", morfología de las palabras.. etc.

Se calificó las pruebas y se sacó la media del grupo para tener una idea sobre la comprensión de lectura sobre el caso clínico para luego contrastar con los resultados del segundo grupo con quienes se hizo la explicación, manejo y uso de las estrategias de lectura y las claves lingüísticas.

De la misma forma se calificó las pruebas del segundo grupo y se contabilizó cuántos estudiantes usaron las estrategias de lectura y las partes lingüísticas para luego tabular los datos y encontrar la frecuencia de uso de las estrategias por parte de los alumnos. De esta manera se pudo evidenciar la causa de la falta de comprensión lectora y cual estrategia es la más y menos usada por parte de los estudiantes.

También se calculó la desviación estándar para ver el grado de utilización de cada pista lingüística por los estudiantes y ver si mantiene un uso regular por todos los estudiantes.

\section{Resultados}

Después de realizar el conteo y tabulación de los datos, se obtuvieron los siguientes resultados de las dos pruebas y con los 2 grupos de estudiantes.

Tabla1.- Media de los dos grupos con y sin utilización de ayudas lingüísticas.

\begin{tabular}{lcc}
\hline & Primer Grupo & Segundo Grupo \\
\hline $\begin{array}{l}\text { Comprensión lectora sin ayudas } \\
\text { lingüísticas y estrategias lectoras }\end{array}$ & 4,79 & \\
$\begin{array}{l}\text { Comprensión lectora con ayudas } \\
\text { lingüísticas y estrategias lectoras }\end{array}$ & 7,04 \\
\hline
\end{tabular}

Fuente: Test de los estudiantes

Elaborado por: Los autores 
En la tabla 1 se muestra el porcentaje de calificaciones alcanzadas sobre 10 por los dos grupos antes y después de la intervención. Se puede ver que el primer grupo tiene una media baja de 4,79 lo cual refleja que la mayoría de alumnos no logran entender las preguntas de la prueba y por ende no hay una comprensión significativa del caso clínico. Mientras que el segundo grupo alcanzó una media de 7,04 con respecto al primero, este cambio considerable obedece a la utilización de las estrategias de lectura y claves lingüísticas socializadas y enseñadas a los alumnos durante las siguientes tres semanas.

Tabla 2.- Media del segundo grupo con las ayudas lingüísticas y la Desviación Estandar

\begin{tabular}{lcc}
\hline & M & DS \\
\hline Asociación de palabras & 7,1 & 0,8 \\
Collocations & 4,6 & 2,9 \\
Morfología de las palabras & 5,4 & 2,2 \\
Gramática & 9,1 & 0,65 \\
Signos de Puntuación & 8,9 & 0,45 \\
Skimming & 7,9 & 0,98 \\
Scanning & 7,4 & 0,85 \\
\hline
\end{tabular}

Fuente: Test de los estudiantes

Elaborado por: Los autores

En la tabla 2, se puede ver el grado de utilización de cada una de las características lingüísticas y las estrategias de lectura como "skimming" y "scanning". Se puede ver en la tabla 2 la frecuencia de utilización de cada característica del idioma para tener una idea de cual domina mas los alumnos y a cual hace falta poner mas atención como punto débil. Hablando de las estrategias de lectura "skimming" y "scanning", estas les permitieron a los alumnos tener una idea general y a la vez específica del texto. Los alumnos demostraron tener un uso apropiado de "skimming" con un 7,9\% que significa leer el texto de corrido sin prestar atención a las palabras desconocidas y muy técnicas con el objetivo de comprender el sentido general del contenido del texto de esta manera comprenden de que se trata el caso clínico; en este caso identificar los síntomas del paciente, y el posible tratamiento a llevarse a cabo. [9]

Una vez identificado la idea principal, los estudiantes son capaces de utilizar la siguiente estrategia lectora, a saber, "scanning" con un $7,4 \%$ que involucra hacer una lectura del 
texto nuevamente prestando atención a las palabras específicas tocante a las enfermedades del corazón o palabras desconocidas que se pueden entender por el contexto del tema o de la oración.

También se evidenció la aplicación de claves lingüísticas como asociar las palabras con un 7,1\%. En el estudio se demostró que a los estudiantes se les hizo mas fácil comprender el caso clínico relacionando las palabras asociadas a enfermedades cardiacas de L2 a L1 como por ejemplo miocardio (myocardial), arritmia (arrhythmia), electrocardiograma (electrocardiogram),.. etc. las cuales tienen un deletreo similar en ambos en Español e Inglès.

También ayudó durante la prueba conocer las "collocations" con 4,6\% relacionados con el tema por ejemplo tomar una aspirina (take a pill), ganar peso (gain weight), desarrollar una ulcera (develop a bedsore),eliminar infecciones (eradicate infections).

En el caso de los estudiantes el saber estas partes de lingüística les ayudó a avanzar rápido con la comprensión del texto.

Con respecto a la morfología de las palabras, de la tabla 2 se puede ver los estudiantes usaron en promedio en un $5,4 \%$.

Entendiéndose morfología como parte de la lingüística que estudia la flexión, la composición, y derivación de las palabras. Se llega a la conclusión que los estudiantes tienen que afianzar su conocimientos de sufijos y prefijos como por ejemplo en prefijos anti-, dis-, in-, un-, pre- y sufijos, -sin, -less, -ful, -ness, -ly, etc. En las tablas 3 y 4 se presentan ejemplos de sufijos y prefijos con palabras médicas.

Tabla 3: Ejemplos de Prefijos

\begin{tabular}{ccc}
\hline Prefijo & Ejemplo & Español \\
\hline Hypo- & hypothyroidism & hipotiroidismo \\
Ecto- & ectopic & ectópico \\
Leuko- & leukocyte & leucocito \\
\hline
\end{tabular}

Fuente: https://studylib.net/doc/8754141/commonly-used-prefixes-and-suffixes-innursing 
Tabla 4: Ejemplos de Sufijos

\begin{tabular}{ccc}
\hline Sufixes & Example & Español \\
\hline -cardia & tachycardia & taquicardia \\
-genic & pathogenic & patógeno \\
-lysis & paralysis & parálisis \\
\hline
\end{tabular}

Fuente: https://globalrph.com/common-medical-terminology-suffix-prefix-and-root-words

Los alumnos en su mayoría alcanzaron una media alta en gramática y puntuación, 9,1\% y $8,9 \%$ respectivamente lo que les permitió tener una mejor comprensión de los tiempos gramaticales si se está hablando en presente, pasado o futuro, o los grados de certeza de las afirmaciones. También no pasar por alto los signos de puntuación como por ejemplo, comas, punto y coma, signos de admiración, signos de pregunta es algo que la mayoría de estudiantes uso como medio para comprender mejor el texto médico.

Finalmente, se hizo el análisis estadístico de la desviación estándar para constatar la dispersión de los datos con respecto a la media de los datos obtenidos y se llega a la conclusión que las características lingüísticas menos usadas por los alumnos son “Collocations" por tener un porcentaje 2,9\% y la morfología de las palabras con 2,2\% lo cual indica que la cantidad de veces que los estudiantes usaron estas características son irregulares, es decir no utilizaron todas las veces que la lectura del caso clínico lo pedía, no así en el caso de las demás ayudas lingüísticas donde se usó en casi todas las oportunidades por los alumnos

\section{Discusión}

Como se ha analizado durante este estudio, el que un texto médico sea entendible para el lector o no va a depender del uso y manejo de estrategias de lectura y características propias del idioma Inglés. Por esa razón, la calidad del texto depende de la disponibilidad de pistas contextuales y las cuales tienen que ver con la presencia de información semántica y lingüística que sea clara y suficiente alrededor de las palabras desconocidas porque aportan pistas al significado de estas cuando se lee un texto.

En tal virtud, el adecuado y comprensible contexto lingüístico en el texto tiene un rol significativo en ayudar a los lectores a llegar al preciso significado de las palabras desconocidas. [11] 
Hay un sinnúmero de estudios realizados sobre la adquisición y el aprendizaje del idioma donde el que aprende usa sus estrategias básicas del primer idioma en este caso español para traducir todas las palabras que va encontrando en el camino para pasar a una etapa donde recurra a un amplio repertorio de estrategias avanzadas donde ya no traduzca las palabras sino las entienda en su mismo contexto del segundo idioma. [12]

De ahí la importancia de enseñar a los alumnos que estudian medicina a avanzar y mejorar su comprensión lectora de los textos y tratados médicos en Inglés a través de estrategias de lectura y características de idioma (language features) para que pasen de una fase temprana de aprendizaje a una avanzada en la comprensión de textos científicos en Inglés.

Meara menciona que los hablantes nativos ya tienen organizado su léxico mental en conexiones semánticas mientras que el que está aprendiendo lo tiene mucho menos establecido [13] razón por la cual los estudiantes universitarios, en especial los estudiantes objeto de este estudio necesitan tener clases donde los profesores de Inglés les ayuden a alcanzar un grado de comprensión lector optimo que les permita profundizar sus investigaciones y seguir adelante con su carrera.

\section{Conclusiones}

- En conclusión, los datos obtenidos de la presente investigación muestra que cuando el profesor socializa y enseña a sus alumnos estrategias en la lectura y a conocer bien el idioma a través de sus características, el alumno va superando sus miedos y bloqueo a aprender Inglés médico y mejora su autoestima y confianza en su aprendizaje del idioma.

- Además, aprende a desarrollar autonomía en su aprendizaje para ya no necesitar de un profesor de Inglés, sino que aplicando las herramientas adquiridas en las aulas vaya llegando a una comprensión cada vez mejor y más profunda de su carrera y así se convierta en un servidor de la salud competente y bilingüe.

\section{Referencias Bibliográficas}

[1].- Bacallao Martínez GC, Aparicio Morales AI, Llanes Alvarez C. Software educativo para la enseñanza de la Propedéutica Clínica y Semiología Médica en idioma inglés. Edumecentro [Internet]. 2016 [citado 2019 Abr 22];8(3):[aprox. 16 p.]. Disponible en: http://revedumecentro.sld.cu/index.php/edumc/article/view/756 
[2].- Bosque Cruz M, Cabrera Albert JS, Ivizate Cruz ME, Echevarría Cabaña G, de la Portilla Negrín R. Strategy to implement the didactic conception of the process of English language training of medical students. Rev Ciencias Médicas [Internet]. 2015 [citado 2019 Abr 22];19(2): 304-19. Disponible en: http://scielo.sld.cu/scielo.php?script=sci_arttext\&pid=S1561$31942015000200014 \& \operatorname{lng}=\mathrm{es}$

[3].- Banguela Guerra PT, Nodarse González NM, Cárdenas Santana J, Concepción Morales Y, Aguilera Cardona A. Compendium of playful activities to teach the English grammar in the second year of Medicine. EDUMECENTRO [Internet]. 2016 [citado 2019 Abr 24];8(1):56-68. Disponible en: http://scielo.sld.cu/scielo.php?script=sci_arttext\&pid=S2077$28742016000100005 \& \operatorname{lng}=\mathrm{es}$

[4].- Rodríguez Ochoa A, Moreno Pupo J, Vázquez Gómez MD, Rodríguez Borges B, Ricardo Mulet Y. Juegos didácticos para el aprendizaje del Inglés en la carrera de medicina. ccm [Internet]. 2015 [citado 2019 Abr 24];19(1):119-27.

Disponible en: http://scielo.sld.cu/scielo.php?script=sci_arttext\&pid=S1560$43812015000100011 \& \operatorname{lng}=\mathrm{es}$

[5].- Harmer, J. (2011). How to Teach English. England: Pearson Longman.

[6]. Gardner, D. 2004. "Vocabulary input through extensive reading: A comparison of words found in children's narrative and expository reading materials?. Applied Linguistics 25/1: 1-37

[7]. Laufer, B. 1992. "How much is lexis necessary for Reading comprehension?" in P. J. L. Arnaud and H. Béjoint (eds.)= Vocabulary and Applied Lingüistics. London: Macmillan. PP. 126-

[8].- Spada, N., \& Lightbown, P. (1993). Instruction and the development of questions in L2 classroom. Studies in second Language acquisition, 15(2), 205-224. doi:10.1017/S0272263100011967

[9].- Silberstein, S. ( 1994). Techniques and Resources in Teaching Reading. New York: Oxford University Press.

[10].- Creswell, J. (2012). Planning, Conducting, and Evaluating Quantitative and Qualitative and Qualitative Research. Pearson. 
[11].-Shiva, K. (2017) The Effect of Contextual Clues and Topic Familiarity on L2 Lexical Inferencing and Retention. Porta Linguarum.

[12].-Hasstrup, K (19) Lexical inference procedures or Talking about words.

[13]. Meara, P. (2000) An improved method of assessing productive vocabulary in an L2. Elsevier 


\section{Para citar el artículo indexado.}

Meza Fiallos, C., Guadalupe Bravo, L., Bravo Montenegro, M., \& Ureña Lara, D. (2019). Estrategias metodológicas para el mejoramiento de la comprensión lectora de los casos
clínicos
en Inglés.
Explorador
Digital,
3(3.1),
255-266.

https://doi.org/10.33262/exploradordigital.v3i3.1.894

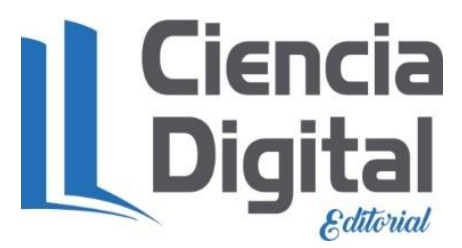

El artículo que se publica es de exclusiva responsabilidad de los autores y no necesariamente reflejan el pensamiento de la Revista Explorador Digital.

El articulo queda en propiedad de la revista y, por tanto, su publicación parcial y/o total en otro medio tiene que ser autorizado por el director o editor de la Revista Explorador

Digital.
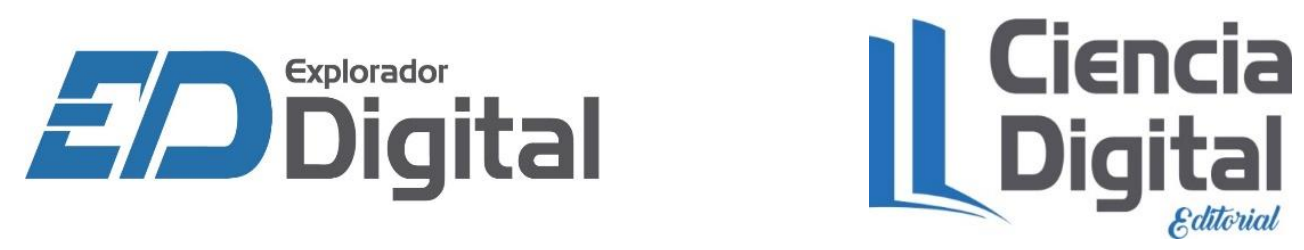